\title{
From NETTER to PETTER: PSMA-Targeted Radioligand Therapy
}

\author{
Matthias Eiber ${ }^{1,2}$ and Ken Herrmann ${ }^{3}$ \\ ${ }^{1}$ Department of Nuclear Medicine, Klinikum Rechts der Isar, Technical University of Munich, Munich, Germany; ${ }^{2}$ Department of \\ Molecular and Medical Pharmacology, David Geffen School of Medicine at UCLA, Los Angeles, California; and ${ }^{3}$ Klinik für \\ Nuklearmedizin, Universitätsklinikum Essen, Essen, Germany
}

$\mathbf{I}_{n}$ et al. present exciting retrospective German multicenter data (12 centers) on the performance of prostate-specific membrane antigen (PSMA)-targeted radioligand therapy (RLT) using ${ }^{177}$ Lu-PSMA 617 in metastatic castration-resistant prostate cancer (mCRPC) (1). The authors describe in detail the safety and efficacy of this new theranostic approach applied under the compassionate use provision in 145 patients.

It took 20 y for somatostatin receptor-directed RLT (also commonly abbreviated as PRRT for peptide receptor RLT) to get close to European Medicines Agency as well as Food and Drug Administration approval (as of today still pending). However, PSMA-directed therapy seems to be off to a promising start. The high demand for PSMA-directed RLT started with the initial report by Zechmann et al. who described the use of a ${ }^{131}$ I-labeled PSMA ligand (2). After

\section{See page 85}

the subsequent introduction of theranostic agents both for imaging and for therapy (e.g., PSMA 617, PSMA I\&T), multiple centers in Germany and worldwide (e.g., Australia, Turkey, and India) adopted this treatment option predominantly using ${ }^{177} \mathrm{Lu}$ as a therapeutic nuclide. Initial clinical experience was followed by multiple relatively small single-institution studies reporting on initial clinical experience with ${ }^{177}$ Lu-PSMA RLT (3-8). However, these studies included a wide range of patients with varying initial and subsequent treatments, different disease stages, and variable study endpoints.

Rahbar's report is the result of a multicenter initiative headed by the German Society of Nuclear Medicine (DGN) that attempts to accelerate the clinical adoption of PSMA-targeted RLT (9). This initiative is highly relevant for patients with advanced disease and is also important for the future of nuclear medicine. Future success will of course depend on a NETTER trial-like

Received Oct. 14, 2016; revision accepted Oct. 19, 2016.

For correspondence or reprints contact: Matthias Eiber, Klinikum Rechts der Isar, Technische Universitat Munchen, Ismaninger Strasse 22, Munich, 81675, Germany.

E-mail: matthias.eiber@gmx.de

Published online Nov. 17, 2016.

COPYRIGHT @ 2017 by the Society of Nuclear Medicine and Molecular Imaging. DOI: 10.2967/jnumed.116.184994 prospective study that paved the way for somatostatin receptortargeted theranostics.

Authors of the DGN consortium summarize experience with 248 treatment cycles in 145 patients. The results are impressive. A $50 \%$ or greater decline in prostate-specific antigen levels occurred in 45\% (45/ 99) of patients in whom follow-up on these values were available. These data are in line with previous smaller studies, some of which are also part of the current report (as described in the supplemental data of Rahbar et al. (1)). Remarkably, the primary endpoint of a $50 \%$ or greater decline in serum prostate-specific antigen levels was achieved already after the first cycle in more than $90 \%$ of responding patients (40/45). Thus, early identification of nonresponders is feasible, allowing for early treatment adaptations in nonresponding patients. In addition, first exploratory data are presented discussing potential negative (visceral metastases, high alkaline phosphatase) and positive (number of cycles applied) response predictors.

The last few years saw the emergence of 5 new drugs for mCRPC. (abiraterone, enzalutamide, sipuleucel-T, cabazitaxel, and ${ }^{223} \mathrm{Ra}$ ) that resulted in some survival benefits (10-14). The data of Rahbar et al. suggest that benefits comparable to those achieved by pharmacologic approaches can be matched or exceeded with ${ }^{177} \mathrm{Lu}$-PSMA RLT. Thus, it could have a major impact on the management of patients with mCRPC. However, larger prospective randomized trials with endpoints including progression-free and overall survival will be needed to determine the precise role among the other emerging therapeutic options.

Given the apparent high efficacy in this heavily pretreated patient group with advanced disease, ${ }^{177}$ Lu-PSMA RLT appears to be well tolerated. Grade 3-4 manageable hematologic toxicity occurred in $12 \%$ of the patients (4\% thrombocytopenia, $10 \%$ anemia). This is comparable to other treatment approaches in advanced mCRPC. A comparable rate of hematologic toxicity was reported in the ALSYMPCA trial $\left({ }^{223} \mathrm{Ra}\right.$-dichloride) (12). Moreover, second-line chemotherapy or radiolabeled antibody therapy is clearly associated with higher rates of toxicity $(13,15)$.

In summary, these German multicenter efforts provided promising preliminary data on effectiveness and tolerability of PSMA-targeted RLT in mCRPC. Our communities (urology, nuclear medicine) are now challenged to avoid the slow translation and acceptance of somatostatin receptor-targeted PRRT. In Germany, this therapy is still applied under compassionate use. Only the recent NETTER-1 trial data have apparently succeeded in finally getting close to market approval and reimbursement in Europe, the United States, and other parts of the world. A PETTER (ProstatE cancer Treatment using endoradioThERapy) trial appears to be the most appropriate response to this challenge. Prospective multicenter randomized trials proving 
the clinical efficacy of ${ }^{177}$ Lu-PSMA RLT heading toward approval and reimbursement are now needed urgently. This is even more challenging because the field of nuclear medicine has struggled in the past quite substantially in conducting multicenter trials. On a more optimistic note, our discipline may translate the lessons learnt in the past and hopefully capitalizes on the knowledge of our clinical partners, which we attempt to summarize as follows.

1. NETTER-1 used available data from clinical experience to initiate a phase 3 study. With regards to ${ }^{177} \mathrm{Lu}$-PSMA, this should help with defining treatment doses and minimize dosimetry requirements. As learnt from NETTER-1, a priori discussions with the regulatory agencies should be helpful.

2. The ALSYMPCA trial taught that overall survival, pain assessment, and skeletal events are important endpoints in the management of mCRPC patients, facilitating approval and reimbursement. However, because various treatment options are available it would be unethical to compare ${ }^{177} \mathrm{Lu}$-PSMA RLT to best supportive care or even placebo. The practical way for study approval and successful enrollment of patients is probably the randomization into 2 groups, both getting medical treatment (e.g., enzalutamide or abiraterone) with 1 group receiving additional ${ }^{177}$ Lu-PSMA RLT. This combination treatment might even enhance the efficacy of ${ }^{177}$ Lu-PSMA because there is a potential upregulation of PSMA expression under hormone ablation (16).

3. The definitions of appropriate clinical endpoints need to be defined in accordance with the recently updated prostate cancer working group 3 framework (17). Despite its well-known limitations, the prostate-specific antigen response after $12 \mathrm{wk}$ remains the key measurement for short-term outcome in all major recent studies. As much as the nuclear medicine community likes to assess the treatment response using theranostic tools (e.g., PSMA PET for treatment monitoring of ${ }^{177}$ Lu-PSMA RLT), these methods have not been validated and are thus not yet been established as objective response parameters. Therefore, trial designs need to use CT and bone scintigraphy criteria, which in prostate cancer are clearly accepted as outcome measurements for progression-free survival. These trials could then be used to add follow-up diagnostic PSMA studies to validate the PET approach as an intermediate endpoint biomarker.

In summary, ${ }^{177} \mathrm{Lu}$-PSMA RLT has the potential to develop into a powerful treatment in mCRPC patients. Its precise position within the growing portfolio of treatment options will need to be established prospectively in well-designed multicenter studies.

\section{REFERENCES}

1. Rahbar K, Ahmadzadehfar H, Kratochwil C, et al. German multicenter study investigating ${ }^{177} \mathrm{Lu}$-PSMA-617 radioligand therapy in advanced prostate cancer patients. J Nucl Med. 2017:58:85-90.
2. Zechmann CM, Afshar-Oromieh A, Armor T, et al. Radiation dosimetry and first therapy results with a ${ }^{124} \mathrm{I} /{ }^{131} \mathrm{I}$-labeled small molecule (MIP-1095) targeting PSMA for prostate cancer therapy. Eur J Nucl Med Mol Imaging. 2014;41:1280-1292.

3. Ferdinandus J, Eppard E, Gartner F, et al. Predictors of response to radioligand therapy of metastatic castrate-resistant prostate cancer with ${ }^{177}$ Lu-PSMA-617. $J$ Nucl Med. September 1, 2016 [Epub ahead of print].

4. Baum RP, Kulkarni HR, Schuchardt C, et al. ${ }^{177}$ Lu-labeled prostate-specific membrane antigen radioligand therapy of metastatic castration-resistant prostate cancer: safety and efficacy. J Nucl Med. 2016;57:1006-1013.

5. Heck MM, Retz M, D'Alessandria C, et al. Systemic radioligand therapy with ${ }^{177} \mathrm{Lu}$ labeled prostate specific membrane antigen ligand for imaging and therapy in patients with metastatic castration resistant prostate cancer. J Urol. 2016; 196:382-391.

6. Rahbar K, Bode A, Weckesser M, et al. Radioligand therapy with ${ }^{177}$ Lu-PSMA617 as a novel therapeutic option in patients with metastatic castration resistant prostate cancer. Clin Nucl Med. 2016;41:522-528.

7. Rahbar K, Schmidt M, Heinzel A, et al. Response and tolerability of a single dose of ${ }^{177}$ Lu-PSMA-617 in patients with metastatic castration-resistant prostate cancer: a multicenter retrospective analysis. J Nucl Med. 2016;57: 1334-1338.

8. Ahmadzadehfar H, Eppard E, Kurpig S, et al. Therapeutic response and side effects of repeated radioligand therapy with ${ }^{177}$ Lu-PSMA-DKFZ-617 of castrateresistant metastatic prostate cancer. Oncotarget. 2016;7:12477-12488.

9. Fendler WP, Kratochwil C, Ahmadzadehfar H, et al. ${ }^{177}$ Lu-PSMA-617 therapy, dosimetry and follow-up in patients with metastatic castration-resistant prostate cancer [in German]. Nuklearmedizin. 2016;55:123-128.

10. Scher HI, Fizazi K, Saad F, et al. Increased survival with enzalutamide in prostate cancer after chemotherapy. N Engl J Med. 2012;367:1187-1197.

11. Ryan CJ, Smith MR, Fizazi K, et al. Abiraterone acetate plus prednisone versus placebo plus prednisone in chemotherapy-naive men with metastatic castrationresistant prostate cancer (COU-AA-302): final overall survival analysis of a randomised, double-blind, placebo-controlled phase 3 study. Lancet Oncol. 2015;16:152-160.

12. Parker C, Nilsson S, Heinrich D, et al. Alpha emitter radium-223 and survival in metastatic prostate cancer. N Engl J Med. 2013;369:213-223.

13. de Bono JS, Oudard S, Ozguroglu M, et al. Prednisone plus cabazitaxel or mitoxantrone for metastatic castration-resistant prostate cancer progressing after docetaxel treatment: a randomised open-label trial. Lancet. 2010;376:11471154 .

14. Huber ML, Haynes L, Parker C, Iversen P. Interdisciplinary critique of sipuleucel-T as immunotherapy in castration-resistant prostate cancer. $J$ Natl Cancer Inst. 2012;104:273-279.

15. Tagawa ST, Milowsky MI, Morris M, et al. Phase II study of Lutetium-177labeled anti-prostate-specific membrane antigen monoclonal antibody J591 for metastatic castration-resistant prostate cancer. Clin Cancer Res. 2013;19:51825191.

16. Bakht MK, Oh SW, Youn H, Cheon GJ, Kwak C, Kang KW. Influence of androgen deprivation therapy on the uptake of PSMA-targeted agents: emerging opportunities and challenges. Nucl Med Mol Imaging. 2016;1-10.

17. Scher HI, Morris MJ, Stadler WM, et al. Trial design and objectives for castrationresistant prostate cancer: updated recommendations from the Prostate Cancer Clinical Trials Working Group 3. J Clin Oncol. 2016;34:1402-1418. 